\title{
Padrões espaciais da Tuberculose e sua associação à condição de vida no município de Ribeirão Preto
}

\author{
Spatial patterns of Tuberculosis and its association with living \\ conditions in the city of Ribeirão Preto in the State of São Paulo
}

\author{
Paula Hino ${ }^{1}$ \\ Tereza Cristina Scatena Villa ${ }^{2}$ \\ Tarcísio Neves da Cunha ${ }^{3}$ \\ Claudia Benedita dos Santos ${ }^{2}$
}

${ }^{1}$ Escola de Enfermagem, Universidade de São Paulo. Av. Dr. Enéas de Carvalho Aguiar 419. 05403-000 São Paulo SP.

paulahino@usp.br

${ }^{2}$ Escola de Enfermagem de Ribeirão Preto, Universidade de São Paulo ${ }^{3}$ Subsecretaria de Vigilância Sanitária, Secretaria Municipal de Saúde/Rio de Janeiro
Abstract This study, with a hybrid, ecological and time-tendency design, sought to establish the relation between the spatial correlation in the occurrence of new cases of TB in the year 2000 and to ascertain the association between living conditions and TB distribution in Ribeirão Preto, State of São Paulo, between 2000 and 2006. The thematic maps were elaborated with the help of MapInfo 7.5 and spatial statistical analysis using Spring 4.3. For the remaining calculations, SPSS 10.0 was used. The indices with the highest factor loads were family heads earning 2 minimum wages or less and with less than 3 years of education. The comparison of the living conditions and TB maps revealed a relation between $T B$ and poorer areas in the city, as the incidence rate in the cluster with poor living conditions was 49.9/100,000 inhabitants. The gross Chance Ratio, considering the cluster with the high living conditions as a reference, proved the association between $T B$ and living conditions and 3.30 for the low living conditions cluster $(C R=3.30$; CI95\%: 1.90-5.70). The city's stratification according to living conditions and incidence of $T B$ allowed for the identification of risk areas, providing input for the local TB Control Program.

Key words Social conditions, Space-time clustering, Epidemiology, Tuberculosis
Resumo Estudo com delineamento híbrido, ecológico e de tendência temporal cujos objetivos foram obter a correlação espacial da ocorrência dos casos novos de Tuberculose ( $\mathrm{Tb}$ ), no período 2000 a 2006 e estudar, no ano de 2000, a associação entre a condição de vida e a distribuição da $\mathrm{Tb}$ para o Município de Ribeirão Preto - São Paulo. Os mapas temáticos foram realizados com auxílio do software MapInfo 7.5 e a análise estatística espacial, por meio do Spring 4.3. Para os demais cálculos, utilizou-se o SPSS 10.0. Os indicadores que apresentaram as maiores cargas fatoriais foram chefes de família com renda menor ou igual a 2 salários mínimos e menos de 3 anos de estudo. A comparação dos mapas de condição de vida e Tuberculose evidenciou relação entre a doença $e$ áreas mais carentes do Município, visto que o coeficiente de incidência no cluster de baixa condição de vida foi de 49,9/100 mil habitantes. A Razão de Chances bruto para o cluster de baixa condição de vida foi igual a 3,30 (RC=3,30; IC 95\%: 1,90-5,70). A estratificação do Município segundo condições de vida e ocorrência da doença nele permitiu a identificação de áreas de risco, fornecendo subsídios para o Programa de Controle da Tuberculose local.

Palavras-chave Condições sociais, Conglomerados espaço-temporais, Epidemiologia, Tuberculose 


\section{Introdução}

As precárias condições de vida a que uma parcela da população está submetida têm repercutido em seus níveis de saúde. No Brasil, assim como em outros países, o processo de desenvolvimento econômico vem se caracterizando, no plano social, por profundas desigualdades, exclusão social e insuficiência de investimentos políticos e financeiros. A desigualdade na distribuição de renda, no acesso aos recursos de saúde, no saneamento básico, na educação e em outros constituintes do padrão de vida da população tem se revelado por meio de diferenças no risco de adoecer dos diversos estratos sociais.

Estudos mostram que a saúde da população apresenta um gradiente social, desfavorecendo os grupos socialmente menos privilegiados, e que a falta de equidade social, no âmbito da saúde, manifesta-se tanto nos diferenciais encontrados nas taxas de morbimortalidade como no adoecimento precoce das camadas menos favorecidas ${ }^{1,2}$. Assim, a construção de indicadores que contribuam para o diagnóstico das condições de vida das populações permite, pela identificação das situações de risco, um direcionamento das ações de saúde de modo mais específico às populações mais vulneráveis.

Em relação à Tuberculose ( $\mathrm{Tb})$, várias pesquisas vêm demonstrando sua relação com as condições de vida, destacando o papel dos indicadores de desigualdade social com a ocorrência da doença ${ }^{3,4}$, cujas populações socialmente desfavorecidas e marginalizadas são atingidas de forma intensa e desproporcional pela $\mathrm{Tb}$, que tem se estabelecido de forma assustadora nos países menos favorecidos ${ }^{5}$.

Assim, diante da situação da Tb no município de Ribeirão Preto torna-se apropriado o estudo da associação entre indicadores pertencentes à esfera social no desenvolvimento do processo da doença, o que pode subsidiar a elaboração de atividades de prevenção e controle da endemia nas áreas que necessitam de maior monitoramento. O conhecimento das condições de vida dos doentes de $\mathrm{Tb}$ e como eles estão distribuídos no espaço geográfico, por meio de técnicas de geoprocessamento, é uma ferramenta que visa a ajudar na elaboração de novas estratégias de controle da doença e também ressaltar a necessidade de superação das iniquidades sociais.

Os objetivos deste estudo foram obter a correlação espacial da ocorrência dos casos novos de Tuberculose, no período 2000 a 2006, e estudar a associação entre a condição de vida e a dis- tribuição da Tb no ano de 2000, no município de Ribeirão Preto, São Paulo.

\section{Métodos}

O município de Ribeirão Preto está situado nas regiões Nordeste do Estado de São Paulo e Sudeste brasileira e, segundo informações do Instituto Brasileiro de Geografia e Estatística (IBGE), em 2006, contava com 559.651 habitantes vivendo em condições urbanas. A área total é de 651 $\mathrm{km} 2$, dividida em 58 subsetores e 651 setores censitários, implicando uma densidade demográfica de 775 habitantes $/ \mathrm{km}^{2}$.

Este estudo caracteriza-se como ecológico e de tendência temporal (retrospectivo e longitudinal). A população compreendeu os casos novos de Tb inscritos no Programa de Controle da Tuberculose (PCT) de Ribeirão Preto no período de 2000 a 2006, segundo o endereço de residência notificado, sendo as informações coletadas na Vigilância Epidemiológica da Secretaria Municipal de Saúde de Ribeirão Preto, por meio do banco de dados Epi-Tb.

Para a descrição da condição de vida, um Indicador Adaptado de Condição de Vida (IACV) foi construído. A seleção das variáveis que compuseram o IACV resultou da revisão da literatura e foram consideradas aquelas que apresentavam tradicionalmente relação com carência social e modo de transmissão da doença. A base de dados do Censo Demográfico de 2000 foi usada para a obtenção das informações socioeconômicas, demográficas e sanitárias sobre a população e os domicílios, desagregados no nível de setor censitário do município de Ribeirão Preto. Por esse motivo, as análises que envolviam o IACV, foram realizadas apenas no ano de 2000. A escolha dos indicadores socioeconômicos baseou-se em estudo realizado no Município de Recife ${ }^{6}$, o qual objetivou caracterizar a mortalidade infantil pela análise das desigualdades no risco de morte e na sua relação com a condição de vida da população. Três variáveis do estudo escolhido como base foram excluídas, sendo as seguintes relacionadas ao saneamento básico: proporção de domicílios com abastecimento adequado de água, domicílios com instalação sanitária adequada e domicílios com coleta direta de lixo, pois o município de Ribeirão Preto apresentou cobertura de quase 100\% para estas variáveis. Diante destas constatações, optou-se por usar as demais variáveis: proporção da população de 10-14 anos analfabeta, proporção de chefes de domicílios com 3 anos ou menos 
de estudo, proporção de chefes de domicílio com renda menor ou igual a 2 salários mínimos e densidade intradormitório.

A opção pela escolha do setor censitário, como unidade de análise, foi em razão deste apresentar a vantagem de ser o nível mais desagregado de grupos populacionais e socioeconômicos, coletados de forma sistemática, periódica e padronizada de abrangência nacional.

Neste estudo, as variáveis consideradas construíram um indicador sintético da condição de vida da população, segundo a Análise Fatorial. Os critérios de seleção adotados para os fatores foram autovalores maiores que um. Os valores do fator extraído (escores fatoriais) foram estimados por regressão. Para a obtenção de estratos de condição de vida, o IACV foi submetido à técnica de agrupamento "hierarchical cluster analysis", na busca de identificar três estratos distintos com condições de vida similares.

Para a geocodificação dos casos, foi usado o software MapInfo 7.5. A base cartográfica digitalizada e georreferenciada de Ribeirão Preto, contendo o arruamento, foi adquirida na Multispectral, e a malha dos setores censitários foi obtida pela loja virtual do IBGE.

Após o georreferenciamento dos casos, foram obtidos padrões de densidade espaciais por meio de análise estatística espacial, segundo a técnica de alisamento Kernel. Para isto, pode-se ajustar uma função bidimensional dos eventos considerados, compondo uma superfície cujo valor será proporcional à intensidade de amostras por unidade de área. Esta função realiza uma contagem de todos os pontos dentro de uma região de influência, ponderando-os pela distância de cada um à localização de interesse ${ }^{7}$.

Especificamente para o ano 2000, foram aplicados o teste Qui-quadrado e a Razão de Chances $(\alpha=0,05)$ e, na etapa de visualização, realizouse a comparação entre o mapa contendo a densidade espacial dos casos de $\mathrm{Tb}$ com o mapa digital de setores censitários estratificados segundo as condições de vida.

Para realização do referido estudo, o projeto foi aprovado em 16 de fevereiro de 2005 pelo Comitê de Ética em Pesquisa envolvendo Seres Humanos da Escola de Enfermagem de Ribeirão Preto da Universidade de São Paulo.

\section{Resultados}

A Figura 1 apresenta o mapa da distribuição de densidade de casos novos de Tb, obtidos segun- do o estimador Kernel, para o período de 2000 a 2006. Vale ressaltar que, apesar do mapa pontual dos casos representar uma forma valiosa de visualização, dado o impacto visual imediato, pode ocorrer uma subestimação dos casos, pois cada ponto não representa necessariamente um caso. Daí, a necessidade de se recorrer à técnica Kernel 7 que permite estimar a intensidade pontual do processo em toda região do estudo. As áreas com taxas mais elevadas estão representadas por tons mais escuros.

Verificou-se uma distribuição desigual da $\mathrm{Tb}$ no município de Ribeirão Preto, onde uma fração considerável dos casos concentrou-se nos bairros das regiões norte e oeste do Município, áreas consideradas mais carentes, e o restante dos casos encontrou-se irregularmente distribuído nos demais bairros. Ressalta-se que, na região oeste, localizam-se as maiores favelas de Ribeirão Preto.

\section{Distribuição espacial da Tb no ano 2000 associada ao Indicador Adaptado de Condição de Vida}

Os dados da Tabela 1 apresentam os valores obtidos para os coeficientes de correlação linear de Pearson entre as quatro variáveis utilizadas para a construção do IACV. Todos os indicadores mostraram-se positivos e estatisticamente correlacionados ( $\mathrm{p}<0,01$ ), com as maiores correlações observadas entre chefes de família com 3 anos ou menos de escolaridade e renda menor ou igual a 2 salários mínimos $(0,84)$, e entre 3 anos de escolaridade e densidade intradormitório $(0,60)$. Em razão das correlações estatisticamente significativas entre as variáveis, considerou-se a existência de um único fator para a composição do IACV, quando se procedeu à análise fatorial, segundo o método de componentes principais. Esse fator foi capaz de explicar aproximadamente $66 \%$ da variação total do conjunto analisado.

Vale ressaltar que, por meio da análise dos valores dos coeficientes de variação, observou-se a presença de extremos na população para os indicadores de chefes de família com renda menor que dois salários mínimos, que variou entre $0 \%$ e $84 \%$ (média de $23 \%$ e CV $=65,2$ ) e chefes de família com menos de 3 anos de estudo, o qual variou entre $0 \%$ e $73 \%$ (média de $17 \%$ e $\mathrm{CV}=64,7$ ).

Em relação à densidade intradormitório, indicador que reflete a densidade intradomiciliar, sobretudo noturna, obteve-se em média 3,4 pessoas por dormitório, variando entre 2 e 5 , sendo o valor mínimo 1,8 pessoas e o máximo 5,2 pes- 


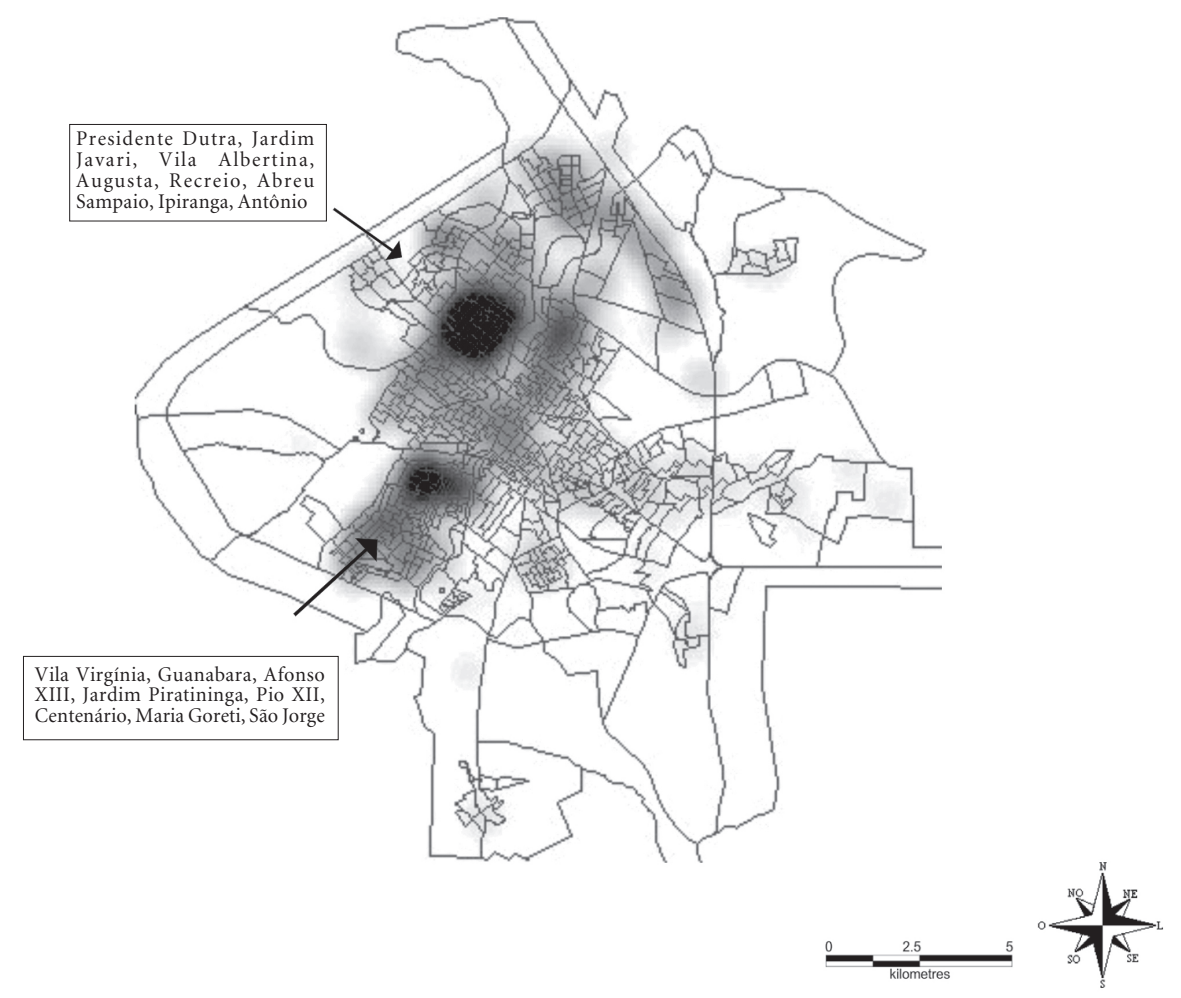

Figura 1. Mapa da distribuição da densidade de casos novos de Tuberculose, obtidos por meio do estimador Kernel. Ribeirão Preto- SP. 2000-2006

Tabela 1. Matriz de correlações das variáveis utilizadas para a construção do Indicador Adaptado de Condição de Vida segundo setores censitários. Ribeirão Preto- SP. 2000

\begin{tabular}{|c|c|c|c|c|}
\hline Variável & 1 & 2 & 3 & 4 \\
\hline $\begin{array}{l}\text { Proporção de chefes de domicílios com renda menor ou igual } \\
\text { a } 2 \text { salários mínimos }\end{array}$ & 1,00 & & & \\
\hline Proporção de chefes de domicílios com 3 anos ou menos de estudo & $0,837^{* *}$ & 1,00 & & \\
\hline Proporção da população de 10-14 anos analfabeta & $0,472^{\star *}$ & $0,387^{\star *}$ & 1,00 & \\
\hline Densidade intradormitório & $0,523^{\star *}$ & $0,598^{\star *}$ & $0,294^{* *}$ & 1,00 \\
\hline
\end{tabular}

Correlações estatisticamente significativas com nível de significância com nível de 0,01

soas. Para a variável proporção da população de 10 a 14 anos analfabeta, o coeficiente de variação foi superior a $100 \%(142,9 \%)$, indicando a ocorrência de extremos aberrantes. Tais resultados expressam a heterogeneidade intraurbana existente no município.

Os dados da Tabela 2 apresentam as cargas fatoriais, que representam a contribuição de cada uma das variáveis secundárias utilizadas na porcentagem de variação explicada e o fator princi- pal que indica condição de vida. Os indicadores com maiores cargas fatoriais foram aqueles relativos às características dos chefes de família: renda $(0,92)$ e estudo $(0,91)$. O indicador menos correlacionado com a condição de vida referiuse à população de 10-14 anos analfabeta $(0,62)$.

Para Ribeirão Preto, observou-se um predomínio dos setores censitários nos clusters de alta e intermediária condições de vida; e 8,2\% representaram o cluster de baixa condição de vida 
(Tabela 3). Como era esperado, os clusters de intermediária e baixa condições de vida apresentaram os maiores Coeficientes de Incidência $(34,4$ casos/100 mil habitantes e 49,9 casos/100 mil habitantes), respectivamente.

Os testes do Qui-quadrado e da Razão de Chances bruto foram calculados segundo o nível socioeconômico. A associação entre a ocorrência da $\mathrm{Tb}$ e a baixa condição de vida foi estatisticamente significativa $\left(\chi^{2} ; 0,05=22,7\right)$. Para o cluster de intermediária condição de vida, obteve-se RC $=2,27$; IC 95\%: $(1,46-3,45)$ e para o de baixa condição de vida RC = 3,30; IC 95\%: (1,90-5,70).

\section{Discussão}

O quadro registrado demonstrou o paradoxo de um município que, apesar de abrigar um dos maiores centros de excelência médica do País, apresenta valores elevados de incidência de $\mathrm{Tb}$, sendo considerado um município prioritário do Estado de São Paulo para o controle da doença. A elevada associação com o Hiv, em torno de $30 \%$, agrava ainda mais a situação da endemia, prejudicando o percentual de cura e elevando o percentual de abandono e mortalidade.

A visualização do mapa de densidade de casos novos de Tb no município, para o período de 2000 a 2006, permitiu uma visão ampliada e possibilitou a identificação de áreas com maior ocor- rência da doença. De modo geral, a análise do mapa temático apontou duas áreas onde se concentraram os casos novos de $\mathrm{Tb}$, compostas por bairros das regiões norte e oeste do município. A ocupação irregular das áreas verdes, que deveriam ser destinadas ao lazer, formam hoje as favelas, consideradas como aglomerado subnormal.

A comparação dos mapas de densidade dos casos de $\mathrm{Tb}$ e de estratificação do município, segundo a condição de vida, mostrou que a distribuição dos casos de Tb em Ribeirão Preto não é uniforme e que as áreas com maior número de casos de Tb coincidem com as áreas de concentração de pobreza e algumas de condição intermediária de vida.

Particularmente para o ano 2000, a obtenção de padrões espaciais da $\mathrm{Tb}$ complementada com a construção do IACV permitiu a identificação de áreas de risco em bairros mais carentes e em alguns pertencentes a estratos de condição intermediária de vida, sugerindo a existência de bolsões de pobreza, distribuídos entre os setores censitários do município.

Os setores censitários que compuseram o cluster considerado como de alta condição de vida, destacaram-se pela baixa ocorrência de casos de $\mathrm{Tb}$, dado que estes setores estão situados em bairros com boa infraestrutura urbana e serviços de saúde. No segundo aglomerado, composto por setores de intermediária condição de vida, encontraram-se casos de $\mathrm{Tb}$ nas regiões norte e leste do

Tabela 2. Matriz de cargas fatoriais das variáveis utilizadas para a construção do Indicador Adaptado de Condição de Vida segundo setores censitários. Ribeirão Preto-SP. 2000

\begin{tabular}{lc}
\hline \multicolumn{1}{c}{ Variáveis } & $\begin{array}{c}\text { Cargas fatoriais } \\
\text { (loadings) }\end{array}$ \\
\hline Proporção de chefes de domicílios com renda menor ou igual a 2 salários mínimos & 0,918 \\
Proporção de chefes de domićlios com 3 anos ou menos de estudo & 0,909 \\
Densidade intradormitório & 0,758 \\
Proporção da população de 10-14 anos analfabeta & 0,619 \\
\hline
\end{tabular}

Correlações estatisticamente significativas com nível de significância com nível de 0,01

Tabela 3. Coeficiente de incidência de Tuberculose segundo cluster, Ribeirão Preto- SP. 2000

\begin{tabular}{lcccc}
\hline $\begin{array}{c}\text { Cluster/ } \\
\text { condição de vida }\end{array}$ & $\begin{array}{c}\text { No setores } \\
\text { censitários }\end{array}$ & $\begin{array}{c}\text { No casos } \\
\text { novos }\end{array}$ & População & $\begin{array}{c}\text { Coeficiente de incidência } \\
(/ \mathbf{1 0 0 . 0 0 0} \text { hab })\end{array}$ \\
\hline Alta & 289 & 29 & 191.554 & 15,1 \\
Intermediária & 290 & 90 & 261.556 & 34,4 \\
Baixa & 53 & 23 & 46.093 & 49,9 \\
\hline
\end{tabular}

\footnotetext{
“41 casos cujos clusters não classificados. * 18 setores censitários não classificados.
} 
município e em alguns setores isolados das regiões oeste e sul. Em relação ao cluster de baixa condição de vida, a distribuição dos setores foi bastante irregular em Ribeirão Preto, sendo na maioria das vezes distante da região central. Este cluster apresentou os piores valores para todos os indicadores considerados no estudo, além de apresentar o maior coeficiente de incidência de Tb.

Os testes Qui-quadrado e Razão de Chances evidenciaram uma associação entre a ocorrência da Tb e a condição de vida no ano 2000. É importante ressaltar que, para a população dos clusters de intermediária e baixa condição de vida, a Razão de Chances foi 1,45, podendo-se atribuir este baixo valor à existência de bolsões de pobreza, encontrados nos setores censitários que fazem parte desses clusters. Portanto, o risco de Tb é quase igual para estes dois clusters.

Quando se discute uma doença como a Tb, deve ser orientada uma visão que vai além das fronteiras da clínica ${ }^{8}$; devem ser envolvidas as condições de vida dos doentes, visto que não é recente a discussão de que a pobreza é o ambiente natural $\mathrm{da} \mathrm{Tb}$. Enquanto o controle da $\mathrm{Tb}$ for considerado apenas como um somatório de doentes que precisam de uma intervenção biomédica, focada no alcance da cura, continuaremos a ter casos de $\mathrm{Tb}$ em nosso meio.

Deste modo, a $\mathrm{Tb}$ deve ser entendida como um processo que se desenvolve em indivíduos que integram uma determinada organização social, estando sujeitos a riscos e potencialidades distintas, segundo seu pertencimento a determinados grupos, visto ser impossível avançar uma proposta sem que haja integração com outros setores do governo para reunir esforços para reduzir a pobreza, a desigualdade e a exclusão social, e que o País deve buscar alternativas para melhor distribuição de renda, redução do desemprego, melhorar o acesso e a qualidade de assistência à saúde, bem como o saneamento básico, entre outras prioridades.

Assim, a proposta da Vigilância da Saúde pode ser considerada um eixo reestruturante da maneira de se agir em saúde, constituindo um esforço para integrar a atuação do setor saúde, considerando as dimensões sociais e os riscos ambientais, epidemiológicos e sanitários. A partir de um diagnóstico da situação de saúde de um dado território, os problemas de saúde passam a ser analisados de forma integrada e conforme as necessidades da população.

A incorporação desse novo olhar para a saúde da população é uma possibilidade real e não uma utopia, pois leva em conta múltiplos fato- res que podem estar envolvidos em torno da doença e ainda propõe o envolvimento do indivíduo e da comunidade na construção de sua saúde. Para isto, as ações de saúde precisam ser repensadas no sentido de desenvolver uma articulação entre as diferentes áreas e não apenas a da saúde que, de forma isolada e desintegrada, não consegue atenuar as disparidades existentes, tampouco alcançar meta alguma.

Para isto, as ações de saúde precisam ser repensadas no sentido de desenvolver uma articulação entre as diferentes áreas e não apenas a da saúde que, de forma isolada e desintegrada, não consegue atenuar as disparidades existentes, tampouco alcançar meta alguma.

\section{Limitações do estudo}

A não geocodificação de $10 \%$ dos casos novos de $\mathrm{Tb}$ sugere a possibilidade de parte destes pertencerem a áreas carentes do município, que apresentam bolsões de pobreza, como favelas e assentamentos. Tal fato pode subestimar a realidade da distribuição da doença no município e, assim, prejudicar o planejamento e o monitoramento de ações específicas.

Em relação à geocodificação dos casos, a qualidade da variável endereço e a eficiência do Sistema de Informação Geográfica (SIG), para localizar os eventos pontualmente, são imprescindíveis para possibilitar as análises dos padrões de distribuição dessas ocorrências. Para tanto, o profissional de saúde deve estar consciente da importância do preenchimento da ficha de notificação compulsória com informações corretas e consistentes, pois será por meio destes dados que serão realizadas consultas que proporcionarão maior conhecimento sobre a doença e fornecerão subsídios na tomada de decisões. É preciso que o profissional não entenda esta atividade como uma função burocrática, mas sim como uma forma de contribuir para o controle da doença ${ }^{9}$.

\section{Considerações finais}

A concepção de espaço que envolve a relação dos grupos sociais pode facilitar a compreensão da $\mathrm{Tb}$, pois embora a $\mathrm{Tb}$ seja transmitida por um bacilo, apresenta também como causa a pobreza, sendo por isto considerada uma doença complexa que envolve diversos condicionantes e não apenas o agente transmissor.

O uso de um SIG permitiu o conhecimento da distribuição espacial da Tb em Ribeirão Preto, 
retratando as desigualdades sociais da doença por meio da sobreposição de mapas com informações socioeconômicas e de $\mathrm{Tb}$, por reconhecer que a distribuição da doença no espaço geográfico não é necessariamente a mesma em todos os grupos da população e que fatores socioeconômicos têm relação com a ocorrência da doença.

A estratificação do município em distintas áreas, segundo as condições de vida e Tb, forneceu uma nova informação que dificilmente seria possível obter por meio de informações tabulares. Os resultados permitiram enxergar o município não mais como um todo, mas sim como uma coleção de subconjuntos heterogêneos que são de fundamental importância para a definição de prioridades das ações de controle da $\mathrm{Tb}$, permitindo assim uma racionalização de recursos.

O reconhecimento das áreas de risco fornece subsídios para o planejamento de ações focalizadas nesses grupos, tanto em relação à necessidade de melhorias sociais como na compreensão do espaço, segundo o olhar da Vigilância da Saúde. Portanto, as políticas de prevenção e de controle da Tb devem considerar as diferentes realidades. A diminuição da Tb em nosso meio não pode ser considerada apenas responsabilidade do setor saúde, pois é reconhecido que a saúde é determinada por processos externos a ela. É necessário mudar também as condições sociais, diminuir a desigualdade e a exclusão social, ressaltando a importância do estabelecimento de ações intersetoriais que possam contribuir para a construção de uma sociedade mais equânime e saudável.

Portanto, é preciso estabelecer se realmente as desigualdades socioeconômicas apresentam relação com a ocorrência da $\mathrm{Tb}$, identificar os segmentos mais vulneráveis, priorizando ações de vigilância à doença na tentativa de se conseguir um maior impacto sobre os grupos populacionais prioritários e, posteriormente, dar seguimento ao processo de planejamento e programação local, visto que as iniquidades sociais nos perfis de $\mathrm{Tb}$ e condições de vida constituem as principais barreiras ao avanço e à melhoria da situação de saúde.

Espera-se que este estudo traga contribuições para o PCT local, na medida que disponibiliza informações até então desconhecidas da distribuição espacial e das condições de vida dos doentes de $\mathrm{Tb}$, apontando áreas que devem ser consideradas prioritárias para o controle da doença.

\section{Colaboradores}

P Hino, TCS Villa, TN Cunha e CB Santos participaram igualmente de todas as etapas da elaboração do artigo. 


\section{Referências}

1. Swarcwald CL, Bastos FI, Esteves MAP, Andrade CLT, Paez MS, Medici EV, Derrico M. Desigualdades de renda e situação de saúde: o caso do Rio de Janeiro. Cad Saude Publica 1999; 15(1):15-28.

2. Serpa IM, Pardo CL, Hernández RA. Un estúdio ecológico sobre tuberculosis en un Município de Cuba. Cad Saude Publica 2003; 19(5):1305-1312.

3. Vicentin G, Santo AH, Carvalho MS. Mortalidade por tuberculose e indicadores sociais no Município do Rio de Janeiro. Cien Saude Colet 2002; 7(2):253-263

4. Souza WV, Albuquerque, MFM, Barcellos CC, Ximenes RAA, Carvalho MS. Tuberculose no Brasil: construção de um sistema de vigilância de base territorial. Rev Saude Publica 2005; 39(1):82-89.

5. Santos MLSG, Vendramini SHF, Gazetta CE, Oliveira SAC, Villa TCS. Poverty: socioeconomic characterization at tuberculosis. Rev Latino-am Enfermagem 2007; 15(número especial): 762-767.

6. Guimarães MJB, Marques NM, Melo Filho DA, Szwarcwald CL. Condição de vida e mortalidade infantil: diferenciais intra-urbanos no Recife, Pernambuco, Brasil. Cad Saude Publica 2003; 19(5):1413-1424.

7. Câmara G, Carvalho MS, Cruz OG, Correa V. Análise espacial de áreas. [livro on-line]. 2001. [acessado 2007 jun 26]. Disponível em: http://www.dpi. inpe.br/gilberto/livro

8. Souza JN, Bertolozzi MR. A vulnerabilidade à tuberculose em trabalhadores de enfermagem em um hospital universitário. Rev Latino-am Enfermagem 2007; 15(2):259-266.

9. Hino P. Padrões espaciais da tuberculose associados ao Indicador Adaptado de Condição de Vida no município de Ribeirão Preto [tese]. Ribeirão Preto: Escola de Enfermagem de Ribeirão Preto da Universidade de São Paulo; 2008.

Artigo apresentado em 19/06/2009

Aprovado em 23/01/2010

Versão final apresentada em 05/02/2010 